\title{
Defining the Roles of Data Manager and Epidemiologist in Emergency Medical Teams
}

\author{
Andrea Bartolucci, PhD; ${ }^{1}$ Anisa JN Jafar, MBChB(e), DTM\&H, MRCP, AFHEA, MRCEM, \\ MPH; ${ }^{1}$ Derek Sloan; ${ }^{2}$ Jimmy Whitworth, MD, FmedSci, FRCP, FFPH, DTM\&H ${ }^{3}$
}

1. Humanitarian and Conflict Response Institute, University of Manchester, Manchester, United Kingdom

2. School of Medicine, University of St Andrews, St Andrews, Scotland

3. Department of Infectious Disease Epidemiology, London School of Hygiene and Tropical Medicine, London, United Kingdom

\section{Correspondence:}

Andrea Bartolucci, $\mathrm{PhD}$

Humanitarian and Conflict Response Institute (HCRI)

University of Manchester

Manchester, United Kingdom

E-mail: and.bart.olucci@gmail.com

Conflicts of interest/funding: This study was funded by the Hong Kong Jockey Club Charity Trust within the collaborative project "Training and Research Development for Emergency Medical Teams with reference to the WHO Global EMTs initiative, classification, and standards" between the Humanitarian and Conflict Response Institute (HCRI; Manchester, United Kingdom) and the Hong Kong Jockey Club Disaster Preparedness and Response Institute (HKJCDPRI; Aberdeen, Hong Kong). The authors have no conflicts of interest to declare.

Keywords: data manager; emergency medical teams; epidemiologist; role description

\section{Abbreviations: \\ DM: data manager \\ EMT: emergency medical team \\ EMTCC: Emergency Medical Team \\ Co-ordinating Cell \\ MDS: Minimum Data Set \\ $\mathrm{MoH}$ : Ministry of Health \\ NGO: nongovernmental organization \\ SOP: Standard Operating Procedure \\ WHO: World Health Organization}

Received: March 29, 2019

Revised: July 4, 2019

Accepted: July 21, 2019

doi:10.1017/S1049023X19004965

\begin{abstract}
Medical and epidemiological documentation in disasters is pivotal: the former for recording patient care and the latter for providing real-time information to the host country. Furthermore, documentation informs post-hoc analysis to improve the effectiveness of future deployments.

Although documentation is considered important and indeed integral to health care response, there are many barriers and challenges. Some of these challenges include: working without well-established standards for medical documentation; and working with international guidelines which provide minimal guidance as to how health data should be managed practically to ensure accuracy and completion. Furthermore, there is a shift in mindset in disaster contexts wherein most health care focus shifts to direct clinical care and diverts almost all attention from quality documentation.

This report distinguishes between the tasks of the epidemiologist and the data manager (DM) in an emergency medical team (EMT) and discusses the importance of data collection in the specific case of an EMT deployment. While combining these roles is sometimes possible if resources are limited, it is better to separate them, as the two are quite distinct. Although there is overlap, to achieve the goals of either role, preferentially they should be carried out by two people working closely together with complementary skill sets. The main objective of this report is to provide guidance and task descriptions to EMTs and field hospitals when training, recruiting, and preparing DMs and epidemiologists to work within their teams. Clear delineation of tasks will lead to better quality data, as it commits DMs to being concerned with the provision of real-time documentation from patient arrival through to compiling daily reports. It also commits epidemiologists to providing enhanced disease surveillance; outbreak investigation; and a source of reliable and actionable information for decision makers and stakeholders in the disaster management cycle.
\end{abstract}

Bartolucci A, Jafar AJN, Sloan D, Whitworth J. Defining the roles of data manager and epidemiologist in emergency medical teams. Prehosp Disaster Med. 2019;34(6):668-674.

\section{Introduction}

Most disasters and many major incidents have significant short- and long-term impacts on population health, placing additional demands on health care organizations. ${ }^{1}$ Following disasters, damaged or destroyed health facilities may be unable to adequately provide health care services to victims, and the high rate of injury worsens the impact and renders response management more complex. ${ }^{2}$ When such events exceed the capacity of the country to cope with its own resources, assistance from external sources is often required. ${ }^{3}$

This international relief is often provided in the form of an emergency medical team (EMT) deployment. These EMTs are groups of health professionals whose goal is to treat patients in a country affected by an emergency or disaster. Following events, such as the earthquake in Haiti in 2010, serious questions were raised about the clinical competence and practice of some of the foreign medical teams (now known as EMTs) who delivered some unacceptable practices during their provision of international medical assistance. ${ }^{4}$ The World Health Organization (WHO; Geneva, Switzerland) EMT verification system, that sets guidelines and minimum standards, ${ }^{5,6}$ was created to ensure greater accountability, quality, and coordination of EMTs. One of the WHO verification requirements is to demonstrate that individual clinical care will be documented and that the team will provide a daily report to the Ministry of Health $(\mathrm{MoH})$ and EMT Coordinating Cell (EMTCC) which provides $\mathrm{WHO}$ support to the host-country co-ordination effort. ${ }^{7}$ 


\section{Data Collection in an EMT}

The objective of medical documentation in disasters is to transparently record the care provided, provide accurate and timely data to the host country, and to allow comprehensive post-deployment analysis to improve the effectiveness of future deployments., All EMT members ought to play a part in this; however, there are some team members and roles whose primary focus involves data generation, collation, and analysis.

\section{Disaster Epidemiology}

The $\mathrm{WHO}^{10}$ defines epidemiology as:

The study of the distribution and determinants of health-related states or events (including disease), and the application of this study to the control of diseases and other health problems.

The term refers to the use of epidemiological methods, such as surveillance and scientific research, to assess and investigate adverse health effects after an event, both short- and long-term, and to anticipate consequences of future events on affected populations. ${ }^{11}$ This definition includes various areas of epidemiology (eg, communicable disease, environmental health, and chronic disease) and uses different methods to assess the scope of public health problems in communities.

Disaster epidemiology (ie, applied epidemiology in disaster settings) presents a source of reliable and actionable information for decision makers and stakeholders in the disaster management cycle. ${ }^{11}$ Disaster epidemiology activities, such as rapid needs assessment and surveillance, assist decision makers by providing situational awareness for characterizing an incident's immediate effects on human health, short- and long-term consequences, and impacts of targeted actions and interventions.

In the context of an EMT deployment, the term "disaster epidemiology" gains a further meaning, which includes collecting of data on coordination, communication, and management skills to develop lessons-learned and to contribute to evidence-based decision making for future events.

Considering the significant surge in medical demand and the variety of services provided by EMTs, gathering information from deployed EMTs and the subsequent data analysis is crucial for coordination and decision making to facilitate a timely response. ${ }^{7}$

\section{The Challenges of Data Collection}

Despite its importance, many countries do not systematically collect disaster-related data and information. ${ }^{12}$ The chaotic and confused nature of a disaster, and simultaneous lack of standardization, training, and awareness, compounds the paucity of quality documentation and any notion of methodologically sound data collection. ${ }^{13}$ Non-systematic data collection will make the collation of information very challenging.

It is fair to state that documentation has the potential to be timeconsuming and to detract from patient care. ${ }^{14}$ In many countries' day-to-day practice, the sheer amount of documentation routinely recorded at every medical encounter has grown dramatically so that it may now occupy between one-quarter to one-half of a clinician's time. ${ }^{14}$ This time may increase even further if staff are not familiar with the environment and documentation processes, and the increased burden will consequently push documentation lower down the list of priorities.

In field hospital facilities, organization of care delivery is an immediate problem. ${ }^{15}$ A major challenge facing EMTs is to impose order on the chaos present and prevent its extension into the medical treatment of patients. ${ }^{16}$ A structured documentation process is an essential component of this, which also provides data to inform effective resource utilization. However, it needs to be acknowledged that data management in such a setting requires planning, allocated personnel, field guidance, and appropriate standardized tools for disease detection, such as the WHO's Early Warning, Alert, and Response System. ${ }^{17}$

Beyond data collection, one issue faced is that EMTs do not routinely share and disseminate their data with other EMTs, researchers, or nongovernmental organizations (NGOs). ${ }^{12}$ The reasons for this are multifactorial; disaster response involves a range of actors (eg WHO, NGOs, MoH, and others) with individual, sometimes competing priorities. There is an expectation that there will be data sharing with the host country; however, data ownership by national authorities and custodianship by international organizations can be difficult to reconcile and the appropriate levels of consent for exchange of clinical information, even in anonymized form, can be hard to define. Administrative and ethical obstacles to data sharing can impede innovation in disaster relief, international research collaboration, and implementation of best practice and cutting-edge technology in EMT operations. The EMTs and the domain of disaster management are heavily practice orientated. Therefore, research and innovation in this field needs to combine the EMT and disaster management expertise with focused and rigorous academic institutions who have the resources to support such work.

\section{WHO EMT Minimum Data Set for Daily Reporting}

The WHO has developed "a package of essential data items for EMT reporting derived from medical records of patients treated by EMTs" called EMT Minimum Data Set (MDS). ${ }^{18}$ The MDS provides an overview of the type and the severity of cases seen, medical resource requirement, on-going needs, and potential outbreaks. It provides support for decision making involved in the coordination and management of the EMT's response. The daily summary section enables the EMTCC and $\mathrm{MoH}$ to gain quick insight into the main indicators of the EMT activity, namely: number of patients, outcomes, and bed count. The needs and risks section aims to report urgent risk factors within the affected community, as well as communicate what support is required for that particular EMT. This standardization is a step in the right direction, however, the process of policing and enforcing these standards beyond the initial step, whereby the WHO verifies an EMT as having baseline capability to deliver the MDS, remains to be defined.

\section{International Guidelines for EMTs}

Thus far, the role of epidemiologist and data manager (DM) within an EMT have not been delineated within international guidance. Reference is made to collection, collation, and reporting of data; however, the process of delivering this is not encapsulated within a role description. The WHO Classification and Minimum Standards for Foreign Medical Teams in SuddenOnset Disasters makes reference to poor data coming from disasters, yet does not reference the need for specific staffing to manage data. ${ }^{5}$ The European Union amendment of the Commission Implementing Decision 2014/762/EU, which outlines preparation and planning for what it refers to as natural and man-made disasters, despite referencing data-handling, does not refer to either the role of an epidemiologist or DM. ${ }^{19}$ The Sphere Project ${ }^{20}$ states that: 


\begin{tabular}{|l|l|}
\hline $\begin{array}{l}\text { Inclusion } \\
\text { Criterion }\end{array}$ & Details \\
\hline Language & Only references written in English were included. \\
\hline Reference & $\begin{array}{l}\text { Incomplete or inaccurate references were not } \\
\text { included. }\end{array}$ \\
\hline Topic & $\begin{array}{l}\text { Document must discuss/describe the tasks of the DM } \\
\text { and/or the Epidemiologist }\end{array}$ \\
\hline
\end{tabular}

Table 1. Details of the Inclusion Criteria Applied to Search Results

Abbreviation: DM, data manager.

The lead agency produces a regular overall health information report, including analysis and interpretation of epidemiological data, as well as a report on the coverage and utilization of the health services.

However, there is no description or detail pertaining to the mechanism or role of whoever is expected to handle and manage this data. Instead, the focus lies with outbreak response and does not consider the acute use of data to manage disaster response more broadly. This problem is amplified when different actors (eg, clinical care teams, EMTCC logistics, and academic epidemiologists) require different information which is hard to provide from a rapidly assembled dataset in the field. If unsatisfactory data collection tools are distributed without relevant buy-in across the response (and consideration of resource capabilities), there is a risk that they will not be used and individual teams will design alternative, but fragmented and inefficient, ways of generating the specific data which they want. ${ }^{21}$

\section{The Role of the Epidemiologist and the DM in an EMT}

Without reference to role descriptions, it is difficult to draw attention to the importance of documentation and data. If the role is unclear and undesignated, it is highly likely that it will either be overlooked or poorly executed. Below is provided a description of the roles of an epidemiologist and a DM in an EMT derived from available literature. The main objective of this is to provide guidance to EMTs and field hospitals when training, recruiting, and preparing DMs and epidemiologists to work within their teams. Having role descriptions does not seek to dictate whether each role could or should be adopted by the same single person or by more than one person; however, it distinguishes the separate tasks required by each role to ensure that adequate attention is paid to specific aspects of both roles.

\section{Literature Review}

A literature review was carried out to collect information about the main tasks related to the role of DM and epidemiologist in case of disaster or humanitarian intervention. The search was performed through Google Scholar (Google, LLC; Mountain View, California USA); the search engine provides not only scientific literature, but also job descriptions and advertisements. The search consisted both of a primary search using keywords and a secondary grey literature search.

The search selected English language literature using each of the following keywords: (1) data manager; (2) epidemiologist; (3) disaster; (4) humanitarian emergency; (5) field hospital; (6) job description; (7) role description; and (8) tasks. Documents containing a combination of these words were selected and reviewed by the authors (for criteria of inclusion, see Table 1).
Selected studies were analyzed by the authors to extract information about the role of the DM and the epidemiologist, with a special focus on disaster and humanitarian crisis intervention.

\section{Report}

A total of 5,620 results were found; 5,597 records were excluded after reviewing the title and after duplicate removal. Of the 23 records meeting the criteria, 13 met all the criteria for the search for a description of tasks and roles (Table 2). The content of these sources contributes to the discussion of both DM and epidemiologist roles in the context of disaster response in the subsequent sections.

\section{The Role of Epidemiologist as a Member of an EMT}

There are several resources to identify the expected role of an epidemiologist more generally in resource-poor deployments provided by NGOs and international organization such as Médecins Sans Frontières (MSF; Geneva, Switzerland) and the WHO; however, the specific role in an EMT is not formally described. There will of course be epidemiologists working outside of designated EMTs, for example for the $\mathrm{WHO}$ and the $\mathrm{MoH}$. However, the aim here is to focus on the role of an epidemiologist working within an EMT. Table 3 lists the roles as derived from the literature review sources, split between four phases. In considering the outlined tasks, the possibility of rapid changes in the health environment and availability of information should be borne in mind; EMT epidemiologists must work systematically while being flexible to shifting priorities and unexpected events. Furthermore, assimilation of often incomplete information is required with much more speed within emergency response to inform overall health response management.

\section{The Role of the DM in EMTs}

The DM is responsible for the systematic collection, storage, and management of individual patient data collected by the EMT staff. They must ensure that the data recording is as complete as possible and that the minimum required clinical data are regularly shared via the agreed health coordination mechanisms. ${ }^{4}$ This role needs to be performed in close collaboration with any EMT information technology support staff and based on a good recording system to ensure uniformity of data, easier reporting, and data-based decision support. ${ }^{22}$ As with the epidemiology function, an important distinction between data management under routine circumstances and in the context of an EMT is the need for data to be collected, curated, and disseminated quickly enough for real-time decision making as an emergency unfolds.

One of the earliest tasks for the DM (in collaboration with the EMT team leader) is to discuss and agree with the EMTCC and $\mathrm{MoH}$ the plan for data handling post-deployment. They need to establish what the expectation will be regarding individual record retention and utilization of any anonymized data. Early discussions and any conflicting views need to be addressed with the assistance of the EMTCC. Once the EMT is operational, the DM is responsible for supervising the collection of medical data using the method and design adopted by the EMT; they are also responsible for training any local staff in any relevant data entry roles, such as patient registration. Ideally, the patient record should follow each patient's journey from the entry point up until the exit point of the hospital. The DM, using a pre-planned strategy, should ensure no data loss through collecting and collating the records. These records will be used to populate the expected daily report required by the WHO, which will include all MDS items. The daily report 


\begin{tabular}{|c|c|c|c|c|c|}
\hline TITLE & AUTHOR/ORGANIZATION & TYPE & DM & EPI & YEAR \\
\hline Public Health Guide for Emergencies & $\begin{array}{l}\text { The Johns Hopkins, } \\
\text { International Federation of } \\
\text { Red Cross and Red } \\
\text { Crescent Societies }\end{array}$ & Textbook & $\mathrm{X}$ & & 2008 \\
\hline Epidemiology for Health Extension Workers & Kebede Y & Job Description & $\mathrm{X}$ & $\mathrm{X}$ & 2018 \\
\hline $\begin{array}{l}\text { Data Manager Job at Wellcome Trust - Career } \\
\text { Opportunity in Kenya Wellcome }\end{array}$ & Wellcome Trust & Job Description & $\mathrm{x}$ & & 2018 \\
\hline Data Manager & University of Maryland & Job Description & $\mathrm{X}$ & & 2018 \\
\hline Epidemiologist & RTI International & Website & & $\mathrm{X}$ & 2018 \\
\hline $\begin{array}{l}\text { 2nd National Symposium on Medical and Public } \\
\text { Health Response to Bioterrorism }\end{array}$ & The Johns Hopkins & \begin{tabular}{|c|}
$\begin{array}{c}\text { Symposium } \\
\text { Information }\end{array}$ \\
\end{tabular} & $\mathrm{X}$ & $\mathrm{X}$ & 2000 \\
\hline $\begin{array}{l}\text { Outbreak Surveillance and Response in } \\
\text { Humanitarian Emergencies }\end{array}$ & WHO & Guidelines & & $\mathrm{X}$ & 2012 \\
\hline Epidemiologist & Médecins Sans Frontières & Job Description & & $\mathrm{X}$ & 2019 \\
\hline Data Management ${ }^{23}$ & UK EMT & \begin{tabular}{|l|} 
Standard \\
Operating \\
Procedure (SOP)
\end{tabular} & $\mathrm{X}$ & $\mathrm{x}$ & 2018 \\
\hline
\end{tabular}

Table 2. Research Selected for Review

Abbreviations: DM, data manager; EMT, emergency medical team; EPI, epidemiologist; WHO, World Health Organization.

\begin{tabular}{|c|c|}
\hline Phase & Tasks \\
\hline \multirow[t]{6}{*}{ Pre-Deployment } & Design and deliver relevant training for the EMT (eg, communicable disease alerts and surveillance) \\
\hline & Develop relevant research protocols \& confirm adequate training and ethical safeguards \\
\hline & Support the development of recording systems (paper/electronic) \\
\hline & Identify data requirements of potential deployments \\
\hline & Develop outbreak/disease surveillance SOPs pertinent to the EMT \\
\hline & $\begin{array}{l}\text { Provide an acute baseline outbreak/communicable disease situational report based on available information } \\
\text { once a deployment is imminent }\end{array}$ \\
\hline \multirow{6}{*}{ During } & Identify further data needs and develop existing systems to support this \\
\hline & Contribute to public health surveillance for specific communicable diseases posing an outbreak threat \\
\hline & $\begin{array}{l}\text { Supervise data collection, ensure the correct use of data collection tools liaising closely with data management, } \\
\text { providing regular feedback on data collection to ensure quality is maintained }\end{array}$ \\
\hline & Contribute to relevant report-writing \\
\hline & $\begin{array}{l}\text { Regular analysis, visualization and interpretation of data which may be presented in several ways (eg, reports, } \\
\text { formal presentations, informal or at internal meetings) }\end{array}$ \\
\hline & Prepare routine epidemiological reports as agreed by EMT senior management \\
\hline \multirow{3}{*}{$\begin{array}{l}\text { In relation to communicable } \\
\text { disease outbreaks (eg, as part } \\
\text { of Early Warning and } \\
\text { Response Network [EWARN]) }\end{array}$} & Participate in preliminary assessment of alerts or suspected disease outbreaks \\
\hline & $\begin{array}{l}\text { Participate in detailed assessments to confirm an outbreak in liaison with external partners with agreement of } \\
\text { EMT senior management }\end{array}$ \\
\hline & $\begin{array}{l}\text { Contribute to the development and refinement of case definitions for outbreak monitoring in liaison with externa } \\
\text { partners }\end{array}$ \\
\hline
\end{tabular}

Table 3. Epidemiologist Tasks within an EMT (continued) 


\begin{tabular}{|c|c|}
\hline Phase & Tasks \\
\hline & Support local partners in case-finding interviews \\
\hline & $\begin{array}{l}\text { Contribute to wider partner discussions around control measures (eg, vaccination campaigns, WASH } \\
\text { interventions, mortality surveys) }\end{array}$ \\
\hline & Develop descriptive and analytical epidemiology \\
\hline & Evaluate control measures \\
\hline & Provide expert input into information campaigns \\
\hline & Immediately notify alerts to surveillance officer, including rumors \\
\hline & Maintain an alert log of notified alerts \\
\hline & Co-ordinate with cluster focal points \\
\hline & Compile EWARN priority diseases data in weekly reporting form \\
\hline & Train relevant staff in EWARN function and activities \\
\hline & Debrief with the medical staff to assess the relief effort and to improve EMT performance \\
\hline & Produce handover documents, final reports and "lessons learned" documents \\
\hline & Produce any relevant outbreak reports and EMT general reports \\
\hline
\end{tabular}

Table 3. (continued). Epidemiologist Tasks within an EMT

Abbreviations: EMT, emergency medical team; MoH, Ministries of Health; SOP, standard operating procedures; WHO, World Health Organization.

should cover a 24-hour period of EMT activity and the DM should ensure that guidance is followed as to what data should be included from patients who are still undergoing care at the time of reporting. Although the WHO does provide some guidance on this, the EMT, via the DM, needs a consistent and standard approach. All data must be stored securely according to the individual EMT Standard Operating Procedure (SOP), likely to include password protection of digital records and locked containers for paper records with designated key holders.

One of the most challenging parts of the DM role will inevitably be engagement and interaction with the clinical team to reinforce the need for accurate documentation. Liaison with the EMT team lead will be crucial, but so also will be involvement of EMT members during non-clinical duty time, so that the process becomes an integral part of everyone's role.

Resources on the DM role and tasks are limited; however, the United Kingdom EMTs have developed a SOP specifically for data management, and this provides a blueprint for the practical tasks of maintaining data quality during a deployment. ${ }^{23}$ Table 4 summarizes the task of the DM according to the time of the intervention (pre, during, and post).

\section{Discussion}

Every EMT has the responsibility to complete a medical record for each patient and report daily to the $\mathrm{MoH}$ and the EMTCC. The role of the DM in this process is essential, especially as it is known very well that securing and maintaining priority status for medical documentation in disasters is difficult. Similarly, the role of the epidemiologist, which will involve liaison beyond the EMT itself into the community and wider co-ordination mechanisms, is integral. Without role descriptors for each of these, it is easy for them to be amalgamated into one. Depending on the context and team resource, it may be that a single person needs to take the responsibility for all tasks of each role, however, the main aims and focus of each role are quite distinct, despite having some crossover (Figure 1 shows the main roles and crossovers). Therefore, EMTs need to be very clear about the tasks they are asking of their staff in these roles. Ideally, the roles ought to be carried out by two people working closely together with complementary skill sets. Deciding to have a single team member covering both roles requires some testing of this capacity in drills and pre-deployment simulation. It may be that a single person can cover both roles, but concurrently, other members of the clinical team are trained to assist during non-clinical duties.

In a two-person model, the primary consideration of working collaboratively would be to occupy the same working space so that familiarization with each other as colleagues is facilitated. At the very start of a deployment, unless there was specific work-load already identified around a local outbreak, the epidemiologist is likely to be more available to assist the DM in setting up a steady state of data collection and collation. It is beneficial for both to understand the premise of one another's roles to be able to provide any required support. The added benefit of this early integration is that the epidemiologist can be planning data analysis very early on and can work with the DM to ensure that the data flow is not going to miss out on any key elements which cannot be recovered if missed at an early stage. The epidemiologist may be able to represent the DM at meetings at the early stages of setting up data collection, if it is felt that there is too much to be done within the field hospital for the DM to go off-site. Conversely, once the data management system is established and familiar, with routines and processes made more concrete, the DM role will likely become more predictable with some trouble-shooting and mainly reminding and encouraging clinical colleagues in their 


\begin{tabular}{|c|c|}
\hline Phase & Tasks \\
\hline \multirow[t]{4}{*}{ Pre-Deployment } & Lead the development and testing of the selected recording system (paper/electronic) \\
\hline & Develop detailed Data Management SOPs \\
\hline & Ensure all equipment for data collection is encompassed within the EMT kit-list \\
\hline & Support the trialing of data management processes via field simulation \\
\hline \multirow{8}{*}{ During } & Consider how/if the EMT with integrate any external agency expectations of joint databases \\
\hline & As the field hospital develops, establish steady-state data management/flow protocols according to SOPs \\
\hline & Identify any data flow issues and work with the team leads to resolve these to avoid data loss \\
\hline & Train and support lay and medical staff in collection of data \\
\hline & $\begin{array}{l}\text { Supervise data filling, ensure the correct use of data collection tools by clinical staff and provide responsive feedback on } \\
\text { poorly completed data }\end{array}$ \\
\hline & $\begin{array}{l}\text { Discuss with the WHO and } \mathrm{MoH} \text { the approval to keep non-patient identifiable record information for post-deployment } \\
\text { analysis }\end{array}$ \\
\hline & Fill in and submit the WHO daily report \\
\hline & Identify further data needs with the $\mathrm{WHO} / \mathrm{MOH}$ and provide data management support as required \\
\hline \multirow[t]{3}{*}{ Post-Deployment } & Organize and manage the data collected including planning for handover \\
\hline & $\begin{array}{l}\text { Debrief with the medical staff highlighting good/improved practice and areas requiring more work with a view to improve } \\
\text { quality of EMT performance in data collection }\end{array}$ \\
\hline & Collate any learning from the deployment and plan alteration to the data plan accordingly \\
\hline
\end{tabular}

Table 4. Data manager tasks within an EMT

Bartolucci ( 2019 Prehospital and Disaster Medicin

Abbreviations: EMT, emergency medical team; MoH, Ministries of Health; SOP, standard operating procedures; WHO, World Health Organization.

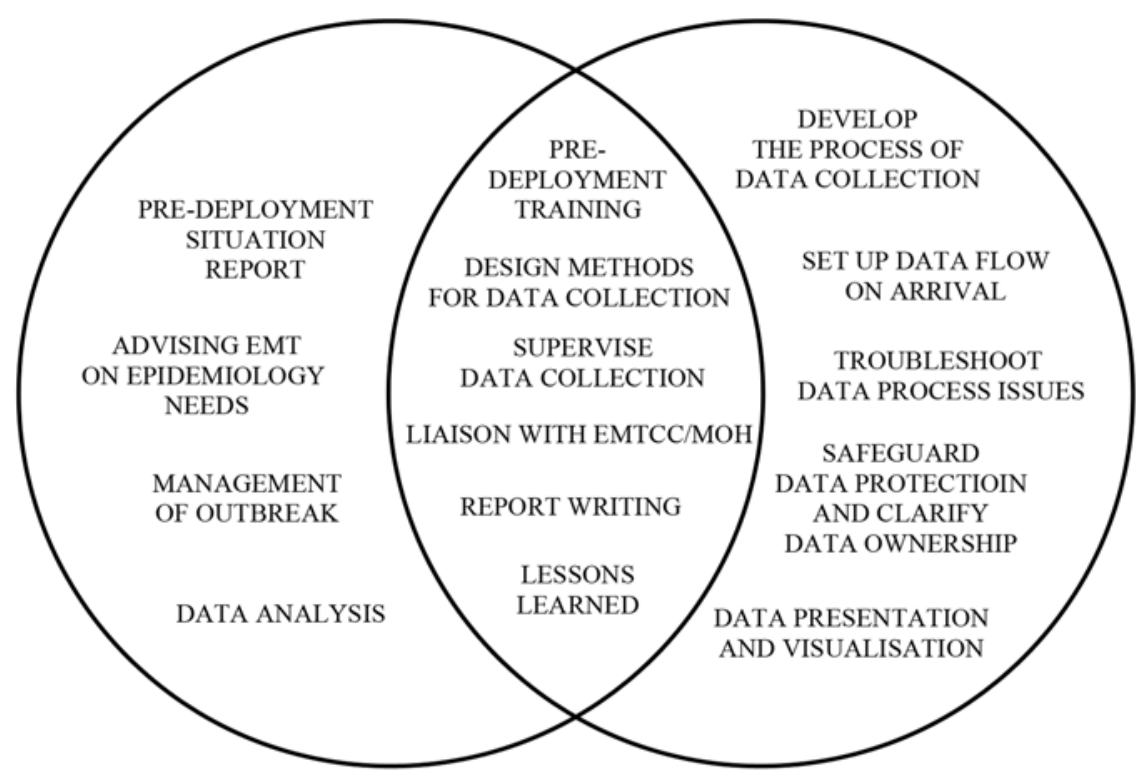

EPIDEMIOLOGIST

DATA MANAGER

miologist Roles and Crossover.

Bartolucci @ 2019 Prehospital and Disaster Medicine

Figure 1. Data Manager and Epidemiologist Roles and Crossover. 
documentation. At this point in time, the deployment will be more established and therefore requirements for the epidemiologist's skills are likely to increase. This is especially true if they are being required to engage with wider outbreak management. The DMs may have the capacity to support the epidemiologist in their role, assisting in aspects of report development, data analysis, and data presentation if appropriate and under the guidance of the epidemiologist.

The key element to this process is planning on the part of the EMT. To leave either role without clarity is likely to result in overlaps and gaps in practice. Therefore, building training around these roles into usual EMT practice will develop familiarity with common pitfalls and lessons learned from past disaster response efforts. This will allow for learning and improvement in practice. Coordination in disaster is essential and relies on information reported by individual teams. Similarly, post-deployment analysis and future improvements hinge on accurate and adequate data.

\section{Conclusion}

This report provides a description of the tasks and responsibilities of the DM and the epidemiologist in an EMT in case of disaster. Just as clinical staff are expected to undergo training specific to field hospital work, DMs and epidemiologists ought to receive specific training for their own roles. It is this groundwork which will pay dividends in the future of disaster response co-ordination, as it integrates focused data training in the EMT pre-deployment plan. In time, this will change the narrative on disaster documentation and data and will allow the international community to confidently understand how best to move forward in medical disaster response.

\section{References}

1. WHO-PAHO. WHO/PAHO Guidelines for the Use of Foreign Field Hospitals in the Aftermath of Sudden-Impact Disasters. Geneva, Switzerland: WHO; 2003.

2. Mäyrä AP, Agapiou A, Hildebrand L, Ojala KM, Mikedi K, Statheropoulos M. Optical sensors for urban search and rescue operations. https://www.spiedigitallibrary.org/ conference-proceedings-of-spie/8185/81850F/Optical-sensors-for-urban-searchand-rescue-operations/10.1117/12.898146.short. Published September 28, 2011. Accessed March 2019.

3. UNISDR. 2009 UNISDR Terminology on Disaster Risk Reduction. https:// www.unisdr.org/files/7817_UNISDRTerminologyEnglish.pdf. Accessed March 2019.

4. Inter-Agency Standing Committee. Coordination and registration of providers of foreign medical teams in the humanitarian response to sudden-onset disasters: a Health Cluster concept paper. https://www.medbox.org/coordination-and-registration-ofproviders-of-foreign-medical-teams-in-the-humanitarian-response-to-sudden-onsetdisasters-a-health-cluster-concept-paper/preview. Published 2012. Accessed March 2019.

5. Norton I, von Schreeb J, Aitken P, Herard P, Lajolo C. Classification and Minimum Standards for Foreign Medical Teams in Sudden Onset Disasters. Geneva, Switzerland: WHO; 2013.

6. WHO. Deploying quality-assured medical teams when disaster strikes. https://www. who.int/hac/donorinfo/2017/deploying-quality-assured-medical-teams/en/. Published February 2, 2017. Accessed March 2019.

7. WHO. Minimum Data Set for reporting by Emergency Medical Teams. http:// docplayer.net/53511352-Minimum-data-set-for-reporting-by-emergency-medicalteams.html. Published 2016. Accessed March 2019.

8. Jafar AJN, Norton I, Lecky F, Redmond AD. A literature review of medical record keeping by foreign medical teams in sudden onset disasters. Prehosp Disaster Med. 2015;30(2):216-222.

9. Mills EJ. Sharing evidence on humanitarian relief. Br Med J. 2005;331(7531):14851486 .

10. WHO. Epidemiology. https://www.who.int/topics/epidemiology/en/. Published 2019. Accessed March 2019.

11. Malilay J, Heumann M, Perrotta D, et al. The role of applied epidemiology methods in the disaster management cycle. Am J Public Health. 2014;104(11):2092-2102.

12. Weichselgartner J, Pigeon P. The role of knowledge in disaster risk reduction. Int J Disaster Risk Sci. 2015;6(2):107-116.

13. Waldman R, Noji EK. Field investigations of natural disasters and complex emergencies. In: Gregg M. Field Epidemiology. Oxford, United Kingdom: Oxford University Press; 2008:472-473.

14. Clynch N, Kellett J. Medical documentation: part of the solution, or part of the problem? A narrative review of the literature on the time spent on and value of medical documentation. Int J Med Inform. 2015;84(4):221-228.

15. Burnweit C, Stylianos S. Disaster response in a pediatric field hospital: lessons learned in Haiti. J Pediatr Surg. 2011;46(6):1131-1139.

16. Levy G, Blumberg N, Kreiss Y, Ash N, Merin O. Application of information technology within a field hospital deployment following the January 2010 Haiti earthquake disaster. J Am Med Informatics Assoc. 2010;17(6):626-630.

17. WHO. Early Warning, Alert, and Response System (EWARS). http://www.who.int/ emergencies/kits/ewars/en/. Published 2018. Accessed March 2019.

18. WHO. EMT MDS Daily Reporting Form. https://www.mdsgateway.net/. Accessed March 2019.

19. ECHO. Amendment of Commission Implementing Decision 2014/762/EU. http:// data.europa.eu/eli/dec_imp1/2014/762/oj. Accessed March 2019.

20. The Sphere Project. The Sphere Handbook: Humanitarian Charter and Minimum Standards in Humanitarian Response. Geneva, Switzerland: Sphere Project; 2011.

21. Polonsky J, Baidjoe A, Kamvar Z, et al. Outbreak Analytics: A Developing Data Science for Informing the Response to Emerging Pathogens. Philos Trans $R$ Soc Lond B Biol Sci. 2019;374(1776):20180276.

22. Bleeker SE, Derksen-Lubsen G, Van Ginneken AM, Van Der Lei J, Moll HA. Structured data entry for narrative data in a broad specialty: patient history and physical examination in pediatrics. BMC Med Inform Decis Mak. 2006;6:29.

23. UKEMT. Data Management. UK EMT Technical Standard Operating Procedure. https://www.who.int/hac/techguidance/tools/standard_operating_procedures_african_ region_en_2014.pdf. Accessed March 2019. 